\title{
一种热活化延迟荧光黄光材料的合成、性能及高效率电致发光器件
}

\author{
王志强 $^{a} \quad$ 蔡佳林 $^{b} \quad$ 张明 ${ }^{b} \quad$ 郑才俊 ${ }^{*, b} \quad$ 吉保明*,a \\ ( ${ }^{a}$ 洛阳师范学院 化学化工学院 河南省功能导向多孔材料重点实验室 洛阳 471934) \\ ${ }^{b}$ 电子科技大学 光电科学与工程学院 成都 610054)
}

\begin{abstract}
摘要 利用吩噁嗪和嘧啶分别作为电子给体和电子受体, 通过 Buchwald-Hartwig 和 Suzuki 偶联反应成功合成了一种热 活化延迟苂光黄光材料 pPBPXZ. 密度泛函理论计算显示, pPBPXZ 分子中吩噁嗪和嘧啶结构单元间的二面角接近 $90^{\circ}$, 而两个嘧啶结构单元与连接二者的苯环间的二面角接近 $0^{\circ} ; \mathrm{pPBPXZ}$ 的最高电子占据轨道主要分布在吩噁嗪结构单元 上, 最低电子未占轨道主要分布在嘧啶环和苯环上, 两种分子轨道只有很小部分重叠. 循环伏安、热重和差热测试表 明, pPBPXZ 具有高的电化学稳定性和热稳定性. 在甲苯溶液中, pPBPXZ 在 360 495 nm 显示出了明显的分子内电荷 转移跃迁吸收, 室温发光峰出现在 $535 \mathrm{~nm}$. 根据低温 $(77 \mathrm{~K})$ 荧光和磷光光谱, 计算得到 pPBPXZ 的最低激发单重态和最 低激发三重态能级分别为 $2.57 \mathrm{eV}$ 和 $2.48 \mathrm{eV}$, 能级差 $\left(\Delta E_{\mathrm{ST}}\right)$ 仅为 $0.09 \mathrm{eV}$. 利用 $\mathrm{pPBPXZ}$ 作为发光层客体掺杂材料, 制备 出了高效率的黄光电致发光器件. 器件的发射峰出现在 $552 \sim 560 \mathrm{~nm}$, 最大电流效率、功率效率和外量子效率分别达到

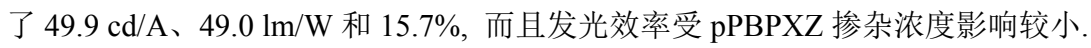

关键词 热活化延迟苂光; 有机电致发光器件; 黄光; 嘧啶; 吩噁嗪

\section{A Novel Yellow Thermally Activated Delayed Fluorescence Emitter For Highly Efficient Organic Light-Emitting Diodes}

\author{
Wang, Zhiqiang $^{a} \quad$ Cai, Jialin $^{b} \quad$ Zhang, Ming $^{b} \quad$ Zheng, Caijun*, $^{*} \quad$ Ji, Baoming*,a \\ ( ${ }^{a}$ College of Chemistry and Chemical Engineering and Henan Key Laboratory of Function-Oriented Porous Materials, \\ Luoyang Normal University, Luoyang 471934) \\ ( ${ }^{b}$ School of Optoelectronic Science and Engineering, University of Electronic Science and Technology of China (UESTC), \\ Chengdu 610054)
}

\begin{abstract}
A novel yellow thermally activated delayed fluorescence emitter pPBPXZ was successfully synthesized using phenoxazine as electron-donor and pyrimidine as electron-acceptor by Buchwald-Hartwig and Suzuki coupling reactions. Density functional theory calculations show that pPBPXZ has highly twisted structure with the dihedrals of nearly $90^{\circ}$ between phenoxazine and pyrimidine units, while the dihedrals between benzene ring and adjacent pyrimidine rings are almost $0^{\circ}$. The highest occupied molecular orbital (HOMO) is mainly confined on two phenoxazine segments, the lowest unoccupied molecular orbital (LUMO) is mainly located on the central pyrimidine and benzene segments, and there is only a slight overlap between HOMO and LUMO. Cyclic voltammetry investigation show pPBPXZ has reversible redox process, and the HOMO and LUMO energy levels were estimated to be $-5.43 \mathrm{eV}$ and $-3.23 \mathrm{eV}$, respectively, from the onsets of oxidation and reduction curves. In diluted toluene solution, pPBPXZ exhibits the absorption band assigned to intramolecular charge-transfer transition and yellow fluorescence with a structureless emission peak at $535 \mathrm{~nm}$. From the onsets of the fluorescence and phosphorescence spectra of pPBPXZ in 2Me-THF at $77 \mathrm{~K}$, the lowest singlet $\left(\mathrm{S}_{1}\right)$ and the lowest triplet $\left(\mathrm{T}_{1}\right)$ energy levels are calculated to be $2.57 \mathrm{eV}$ and $2.48 \mathrm{eV}$, respectively, and thus $\Delta E_{\mathrm{ST}}$ is only $0.09 \mathrm{eV}$. The doped electroluminescence devices using pPBPXZ as guest emitter were prepared by vacuum evaporation method. These devices with doping ratios $(w)$ of $6 \%, 11 \%, 16 \%$ and $23 \%$ show yellow emission at $552 \sim 560 \mathrm{~nm}$ and low turn-on voltages of $3.1 \sim 3.3 \mathrm{~V}$. The device with a doping ratio of $11 \%$ exhibits the highest maximum forward-viewing efficiencies of a maximum current efficiency of $49.9 \mathrm{~cd} / \mathrm{A}$, a maximum power efficiency of $49.0 \mathrm{~lm} / \mathrm{W}$ and a maximum external quantum efficiency of $15.7 \%$ without any light out-coupling enhancement. Particularly, the efficiencies of these devices are not sensitive to the doping ratios of pPBPXZ, which would benefit the further practical application.
\end{abstract}

Keywords thermally activated delayed fluorescence; organic light-emitting diodes; yellow emission; pyrimidine; phenoxazine

* E-mail: zhengcaijun@uestc.edu.cn; lyhxxjbm@126.com

Received October 18, 2018; published December 20, 2018

Supporting information for this article is available free of charge via the Internet at http://sioc-journal.cn.

Project supported by the National Natural Science Foundation of China (No. 51773029) and Henan Natural Science Foundation (No. 182300410230).

项目受国家自然科学基金(No. 51773029)和河南省自然科学基金(No. 182300410230)资助. 


\section{1 引言}

有机电致发光器件(OLEDs)具有驱动电压低、响应 速度快、视角宽、亮度高、色彩鲜艳、轻薄、柔性好等 诸多优点, 作为下一代平板显示和固态照明技术受到了 广泛关注 ${ }^{[10]}$. 苂光材料是最早在 OLEDs 中应用的发 光材料, 但荧光 OLEDs 只能利用电激发产生的单重态 激子发光, 基于该类发光材料的 OLEDs 最大理论内量 子效率仅为 $25 \%$. 铱、铂等磷光过渡金属配合物为第二 代 OLEDs 发光材料. 重金属离子能产生明显的自旋-轨 道耦合, 导致激发三重态和激发单重态混合, 使激发三 重态返回到基态的跃迁不再受自旋禁阻限制, 从而产生 室温磷光; 因此, 磷光 OLEDs 理论上能够将电激发产 生的单重态和三重态激子全部转化为可见光, 最大内量 子效率为 $100 \%{ }^{[11 \sim 13]}$. 然而, 制备高发光效率磷光材料 所需要的金属价格昂贵、资源有限、毒性较大，限制了 过渡金属配合物磷光材料在 OLEDs 中的应用.

近年来, 人们对可利用三重态激子的纯有机发光材 料进行了探索, 包括热活化延迟苂光(TADF)材料、三重 态-三重态湮灭(TTA)材料以及局域电荷转移杂化激发 态(HLCT)材料等 ${ }^{[14 ~ 23]}$. 其中, TADF 材料发展尤为迅速, 已成为 OLEDs 领域的研究热点 ${ }^{[24 ~ 31]}$. TADF 材料一般为 给体-受体(D-A)型分子结构, 材料的最高电子占据轨道 (HOMO) 和最低电子未占轨道(LUMO)分别位于电子给 体和电子受体上, 两种轨道重叠较少; 这种结构使得 TADF 材料的最低激发单重态 $\left(\mathrm{S}_{1}\right)$ 和最低激发三重态 $\left(\mathrm{T}_{1}\right)$ 间能级差 $\left(\Delta E_{\mathrm{ST}}\right.$ ) 较小 (一般小于 $\left.0.3 \mathrm{eV}\right)$, 材料从环境中 吸收的热能可以将 $\mathrm{T}_{1}$ 激子经过反系间窝跃(RISC)过程 转化为 $\mathrm{S}_{1}$ 激子, 再通过辐射跃迁产生苂光 ${ }^{[32 \sim 34]}$. 因此, 基于 TADF 材料的 OLEDs 也能够将电激发产生的单重 态和三重态激子全部转化为可见光.

黄光材料不仅能扩大 OLEDs 的色域, 使显示效果 更自然、逼真, 而且能联合蓝光材料制备白光 OLEDs, 在固态照明领域具有重要应用价值 ${ }^{[35 \sim 43]}$. 本文以吩噁 嗪和嘧啶分别作为电子给体和电子受体, 设计、合成了 一种黄光 TADF 材料 pPBPXZ. 该材料的 $\Delta E_{\mathrm{ST}}$ 仅为 0.09 $\mathrm{eV}$, 发射光具有明显的 TADF 特征. 利用 pPBPXZ 作为 客体掺杂材料制备出了高效率的黄光 OLEDs $\left(\lambda_{\text {max }}=\right.$ $552 \sim 560 \mathrm{~nm}$ ), 器件的最大电流效率(CE)、功率效率 $(\mathrm{PE})$ 和外量子效率 $(\mathrm{EQE})$ 分别达到了 $49.9 \mathrm{~cd} / \mathrm{A} 、 49.0$ $\mathrm{lm} / \mathrm{W}$ 和 $15.7 \%$, 而且发光效率受 $\mathrm{pPBPXZ}$ 的掺杂浓度影 响较小.

\section{2 结果与讨论}

\section{1 合成与结构}

目标化合物 pPBPXZ 的合成路线如图式 1 所示. 以 2 -氯-5-碘嘧啶 (1) 与吩噁嗪 (2) 作为起始原料, 利用二者 间的 Buchwald-Hartwig 偶联反应合成中间产物 3; 化合
物 3 再与对苯二硼酸(4)发生 Suzuki 偶联反应, 即得到 pPBPXZ. 目标化合物 pPBPXZ 的结构通过 ${ }^{1} \mathrm{H}$ NMR、 ${ }^{13} \mathrm{C} N M R$ 以及质谱进行了表征. 在测试物理性能和制备 器件前, 我们用升华法对 pPBPXZ 做了进一步纯化.

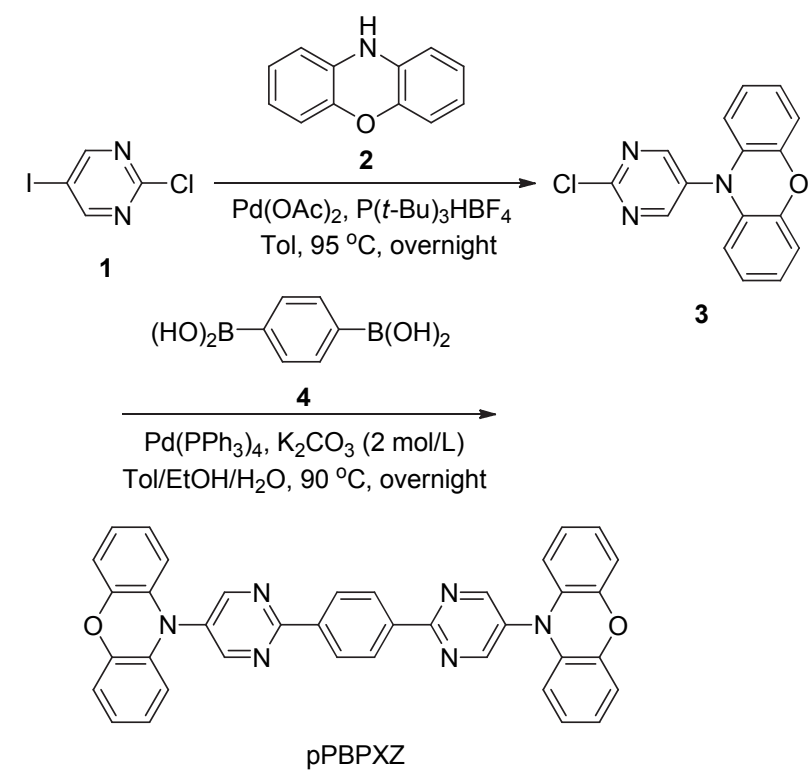

图式 1 化合物 pPBPXZ 的合成路线

Scheme 1 The synthetic route of pPBPXZ

利用 Gaussian09 程序和 B3LYP 方法, 对 pPBPXZ 进行了密度泛函理论 (DFT) 和含时密度泛函理论 (TD-DFT)计算, $\mathrm{C} 、 \mathrm{H} 、 \mathrm{~N} 、 \mathrm{O}$ 原子均用 6-31G*基组. DFT 计算得到的pPBPXZ 立体结构和前沿分子轨道如图 1 所 示. pPBPXZ 中心的苯环与相邻的两个嘧啶环间的二面 角接近 $0^{\circ}$, 三者构成一个 $\pi$ 共轭体系; 而吩噁嗪结构单 元与嘧啶结构单元几乎互相垂直, 之间的二面角为 89.9 . pPBPXZ 的 HOMO 主要分布在吩噁嗪结构单元 上, LUMO 分别主要分布在嘧啶环和苯环上; 通过 TD-DFT 计算得到 pPBPXZ 的 $\mathrm{S}_{1}$ 和 $\mathrm{T}_{1}$ 能级分别为 2.18 和 $2.17 \mathrm{eV}, \Delta E_{\mathrm{ST}}$ 仅为 $0.01 \mathrm{eV}$. 相互分离的 HOMO、

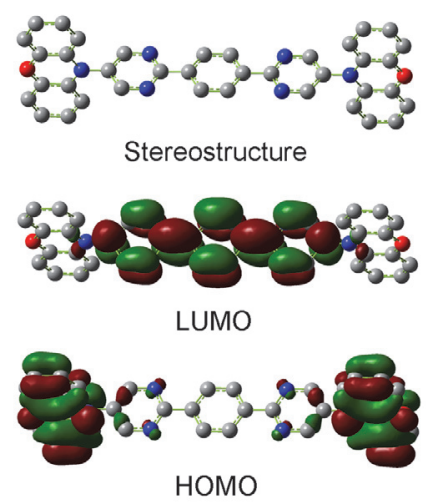

图 1 化合物 pPBPXZ 的立体结构和分子轨道

Figure 1 The stereostructure and molecular orbitals of pPBPXZ 
LUMO 和非常小的 $\Delta E_{\mathrm{ST}}$ 意味着 $\mathrm{pPBPXZ}$ 可能是一种高 效率的 TADF 材料.

\section{2 电化学性能与热稳定性}

循环伏安 $(\mathrm{CV})$ 测试结果显示, pPBPXZ 的电化学氧 化、还原过程均可逆(图 2), 说明该化合物具有良好的电 化学稳定性. 根据起始氧化电位计算得到 pPBPXZ 的 HOMO 能级为 $-5.43 \mathrm{eV}$; 结合 pPBPXZ 吸收光谱的边 沿波长 $(543 \mathrm{~nm})$ 计算出 LUMO 能级为 $-3.15 \mathrm{eV}$, 这一结 果与根据还原电位计算得到的 LUMO 能级 $(-3.23 \mathrm{eV})$ 基本相当; HOMO 与 $\mathrm{LUMO}$ 间的能级差 $(2.28 \mathrm{eV})$ 对应于 黄色发射光. 热重分析(TGA)表明, pPBPXZ 具有高的热 稳定性, 在氮气环境下的热分解温度 $\left(T_{\mathrm{d}}\right.$, 失重 $\left.5 \%\right)$ 达到 了 $451{ }^{\circ} \mathrm{C}$ (图 S1). 在 pPBPXZ 的差示扫描量热(DSC) 测试中, 没有观察到玻璃化转变现象, 说明该化合物具 有良好的形貌稳定性. 高的电化学稳定性和热稳定性有 助于提高电致发光器件的操作稳定性和寿命.

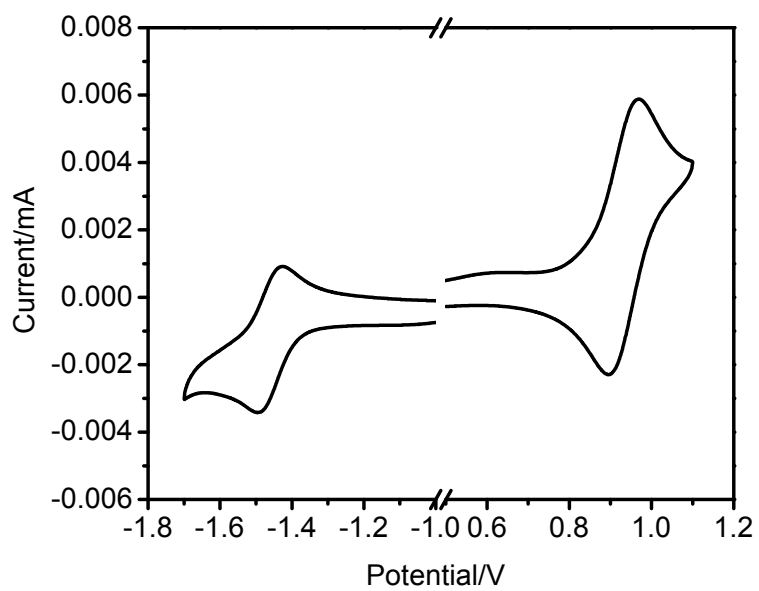

图 2 化合物 $\mathrm{pPBPXZ}$ 的 $\mathrm{CV}$ 曲线

Figure 2 The CV curve of pPBPXZ

\section{3 光物理性能}

化合物 pPBPXZ 在甲苯溶液中、室温条件下的紫 外-可见吸收光谱和发射光谱如图 3 所示. 在吸收光谱 中, 波长小于 $360 \mathrm{~nm}$ 的高强度吸收带 $\left(\lambda_{\text {max }}=320 \mathrm{~nm}\right)$ 为 $\pi-\pi$ *跃迁吸收; $\lambda_{\text {max }}=419 \mathrm{~nm}$ 的吸收带可归属为从电子 给体吩噁嗪到电子受体嘧啶的分子内电荷转移(ICT) 跃 迁. pPBPXZ 在甲苯溶液中发射黄色光, 发光峰出现在 $535 \mathrm{~nm}$. 根据 $77 \mathrm{~K}$ 温度下苂光和磷光光谱的起始波长 (图 S2), 计算得到 pPBPXZ 的 $\mathrm{S}_{1}$ 和 $\mathrm{T}_{1}$ 能级分别为 2.57 $\mathrm{eV}$ 和 $2.48 \mathrm{eV}$, 可见 $\mathrm{pPBPXZ}$ 的 $\Delta E_{\mathrm{ST}}$ 仅为 $0.09 \mathrm{eV}$, 如此 小的能级差意味着该化合物的室温发射光中含有 TADF 组分. 氧气猝灭实验显示, 溶剂中的氧能大幅度降低 pPBPXZ 的发光强度(图 S3), 这说明激发三重态对 pPBPXZ 的发射光有较大贡献. 为了证实 TADF 的存在, 我们将 pPBPXZ 以 $11 \%$ 质量浓度 $(w)$ 掺杂在 4,4'-二(9-咔 唑)联苯 $(\mathrm{CBP})$ 薄膜中, 测试了其在氮气环境中的瞬态苂 光衰减. 结果显示, $\mathrm{pPBPXZ}$ 的室温发射光中含有纳秒
级(9.24 ns) 和微秒级(21.62 $\mu \mathrm{s})$ 两种寿命的发光组分; 其 中, 短寿命组分对应于瞬时苂光，而长寿命组分即为来 源于三重态激子的延迟苂光. 随测试温度的升高, pPBPXZ 的延迟苂光组分明显增强(图 4), 这证实了 pPBPXZ 的延迟苂光为 TADF, 而不是 TTA. 表 1 中为 pPBPXZ 的各项光物理性能数据.

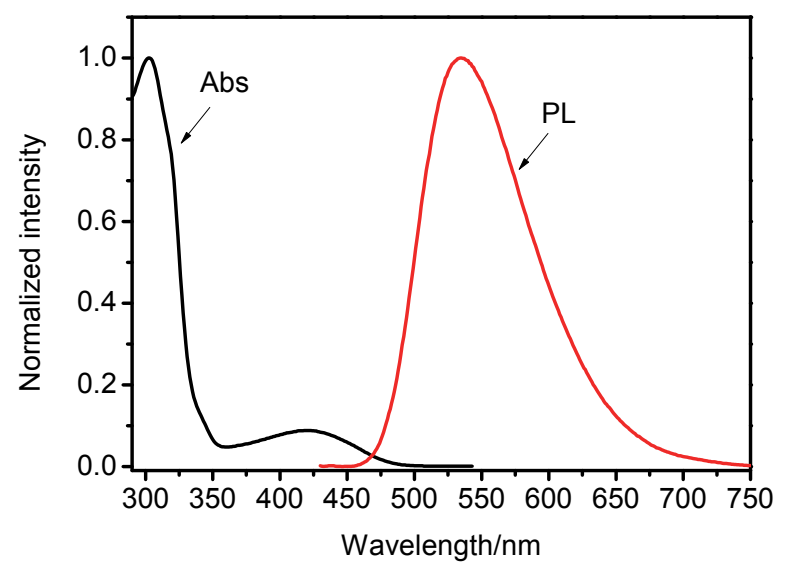

图 3 化合物 pPBPXZ 在甲苯溶液中的紫外吸收与光致发光光谱 Figure 3 The absorption and photoluminescence (PL) spectra of pPBPXZ in toluene solution

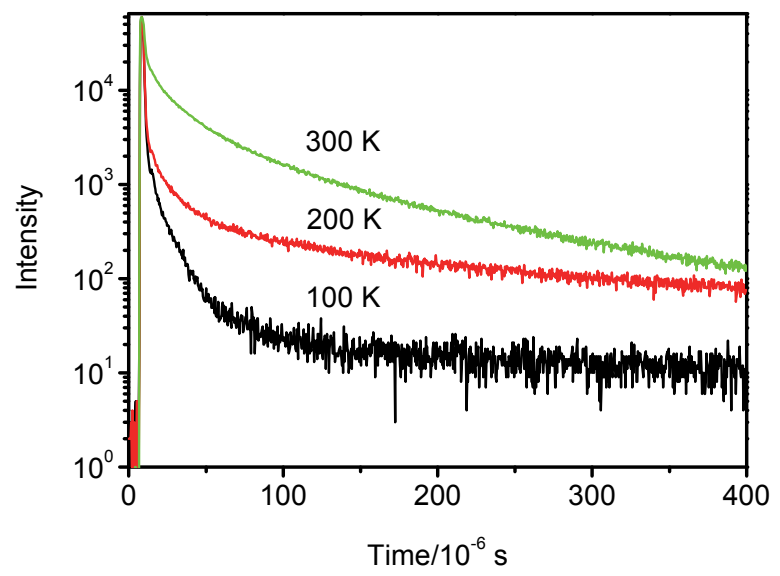

图 4 化合物 pPBPXZ 在 CBP 薄膜中不同温度下的荧光衰减曲线(激 发波长为 $300 \mathrm{~nm}$, 测试波长为 $535 \mathrm{~nm}$ )

Figure 4 The temperature-dependent transient PL decay curves of pPBPXZ in CBP film (Excitation wavelength was $300 \mathrm{~nm}$ and measurement wavelength was $535 \mathrm{~nm}$ )

表 1 pPBPXZ 的光物理性能数据

Table 1 The photophysical data of pPBPXZ

\begin{tabular}{ccccccccc}
\hline $\begin{array}{c}\lambda_{\mathrm{Abs}} / \\
\mathrm{nm}\end{array}$ & $\begin{array}{c}\lambda_{\mathrm{PL}} / \\
\mathrm{nm}\end{array}$ & $\begin{array}{c}\mathrm{S}_{\mathrm{l}} / \\
\mathrm{eV}\end{array}$ & $\begin{array}{c}\mathrm{T}_{1} / \\
\mathrm{eV}\end{array}$ & $\begin{array}{c}\Delta E_{\mathrm{ST}} / \\
\mathrm{eV}\end{array}$ & $\begin{array}{c}\mathrm{HOMO} / \\
\mathrm{eV}\end{array}$ & $\begin{array}{c}\mathrm{LUMO} / \\
\mathrm{eV}\end{array}$ & $\begin{array}{c}\tau_{\mathrm{p}}{ }^{a} / \\
\mathrm{ns}\end{array}$ & $\begin{array}{c}\tau_{\mathrm{d}}{ }^{b} / \\
\mu \mathrm{s}\end{array}$ \\
\hline 419 & 535 & 2.57 & 2.48 & 0.09 & -5.43 & -3.15 & 9.24 & 21.62
\end{tabular}

${ }^{a}$ Lifetime of prompt fluorescence; ${ }^{b}$ Lifetime of delayed fluorescence.

\section{4 电致发光性能}

利用 $\mathrm{pPBPXZ}$ 作为发光层客体掺杂材料, 制备了结 构为 ITO/TAPC (35 nm)/TCTA (10 nm)/CBP:pPBPXZ (20 $\mathrm{nm}) / \mathrm{TmPyPb}(40 \mathrm{~nm}) / \mathrm{LiF}(1 \mathrm{~nm}) / \mathrm{Al}$ 的四种电致发光器 
件. ITO(氧化铟锡)和 $\mathrm{LiF} / \mathrm{Al}$ 分别为阳极和阴极; TAPC、 TCTA、 TmPyPb 和 CBP 分别用作空穴传输层、电子阻 挡层、电子传输层和发光层主体材料, 材料结构如图 5 所示. 在器件 I、II、III、IV 中, pPBPXZ 的质量掺杂浓 度 $(w)$ 分别为 $6 \% 、 11 \% 、 16 \% 、 23 \%$.

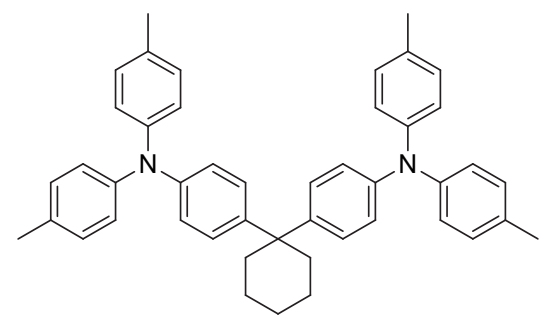

TAPC<smiles>c1cncc(-c2cccc(-c3cc(-c4cccc(-c5cccnc5)c4)cc(-c4cccc(-c5cccnc5)c4)c3)c2)c1</smiles>

$\mathrm{TmPyPb}$

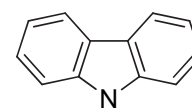<smiles>Nc1ccc(-c2ccc(-n3c4ccccc4c4ccccc43)cc2)cc1</smiles>

$\mathrm{CBP}$

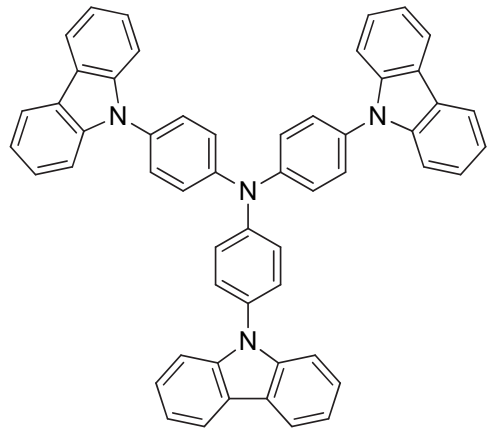

TCTA

图 5 TAPC、TCTA、 TmPyPb 和 $\mathrm{CBP}$ 的分子结构

Figure 5 The molecular structures of TAPC, TCTA, TmPyPb and CBP

表 2 列出了以 $\mathrm{pPBPXZ}$ 作为发光层客体材料的四种 电致发光(EL)器件的主要性能数据. 如图 6 所示, 将 $\mathrm{pPBPXZ}$ 掺杂浓度从 $6 \%$ 提高至 $23 \%$, 电致发光光谱仅 发生了轻微的红移, 器件 I、II、III、IV 的发射峰分别位 于 $552 \mathrm{~nm} 、 552 \mathrm{~nm} 、 556 \mathrm{~nm}$ 和 $560 \mathrm{~nm}$, 该红移应是由 pPBPXZ 分子间的相互作用引起的. 四种器件都具有较 低的开启电压 $\left(V_{\mathrm{on}}=3.1 \sim 3.3 \mathrm{~V}\right)$, 且开启电压随 pPBPXZ 掺杂浓度的增加而降低(图 7). 制备的四种器件 均表现出了高发光效率, pPBPXZ 掺杂浓度为 $11 \%$ 时, 器件 $\mathrm{EL}$ 效率最高, 最大电流效率 $(\mathrm{CE})$ 、最大功率效率
$(\mathrm{PE})$ 和最大外量子效率(EQE) 分别达到了 $49.9 \mathrm{~cd} / \mathrm{A} 、 49.0$ $\mathrm{lm} / \mathrm{W}$ 和 $15.7 \%$. 而且, 器件发光效率对 $\mathrm{pPBPXZ}$ 掺杂浓 度不敏感，尤其是在 $6 \% \sim 16 \%$ 浓度范围内，效率受 pPBPXZ 掺杂浓度影响很小(图 8), 这对材料的实际应用 非常有利.

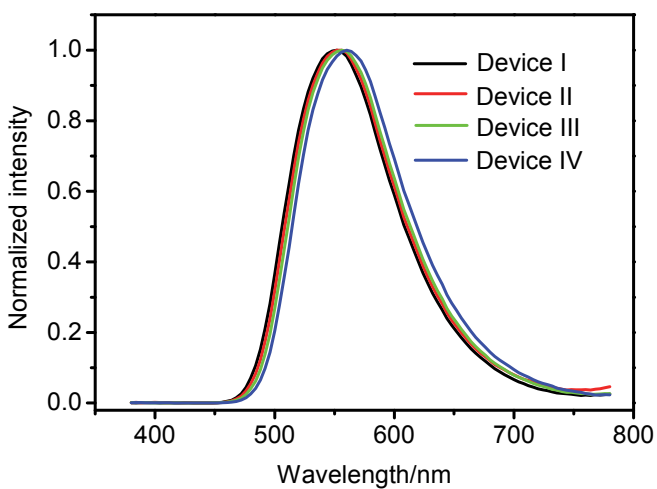

图 6 器件 I、II、III 和 IV 的电致发光光谱

Figure 6 The electroluminescence spectra of devices I, II, III and IV

表 2 器件 I、II、III 和 IV 的电致发光性能数据

Table 2 The EL performance data of devices I, II, III and IV

\begin{tabular}{lcccccc}
\hline Device & $\begin{array}{c}V_{\mathrm{on}}{ }^{a} / \\
\mathrm{V}\end{array}$ & $\begin{array}{c}\lambda_{\mathrm{EL}} / \\
\mathrm{nm}\end{array}$ & $\mathrm{CIE}_{x, y}$ & $\begin{array}{c}\mathrm{CE}_{\max } / \\
\left(\mathrm{cd} \cdot \mathrm{A}^{-1}\right)\end{array}$ & $\begin{array}{c}\mathrm{PE}_{\max } / \\
\left(\mathrm{lm} \cdot \mathrm{W}^{-1}\right)\end{array}$ & $\begin{array}{c}\mathrm{EQE}_{\max } / \\
\%\end{array}$ \\
\hline I & 3.3 & 552 & $(0.40,0.56)$ & 49.6 & 47.4 & 15.5 \\
II & 3.2 & 552 & $(0.41,0.56)$ & 49.9 & 49.0 & 15.7 \\
III & 3.2 & 556 & $(0.42,0.55)$ & 46.2 & 44.6 & 15.1 \\
IV & 3.1 & 560 & $(0.44,0.54)$ & 43.1 & 42.3 & 14.1 \\
\hline
\end{tabular}

${ }^{a}$ Turn-on voltage measured at the luminance of $1 \mathrm{~cd} / \mathrm{m}^{2}$.

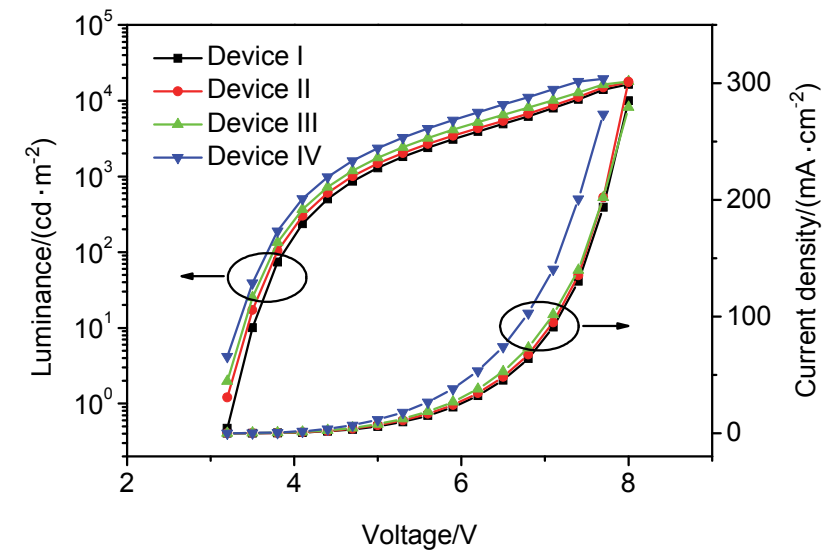

图 7 器件 I、II、III 和 IV 的电流密度-电压-亮度曲线

Figure 7 The current density-voltage-luminance characteristics of devices I, II, III and IV

\section{3 结论}

利用吩噁嗪和嘧啶分别作为电子给体和电子受体, 成功合成了一种新的黄光 TADF 材料 pPBPXZ，该化合 


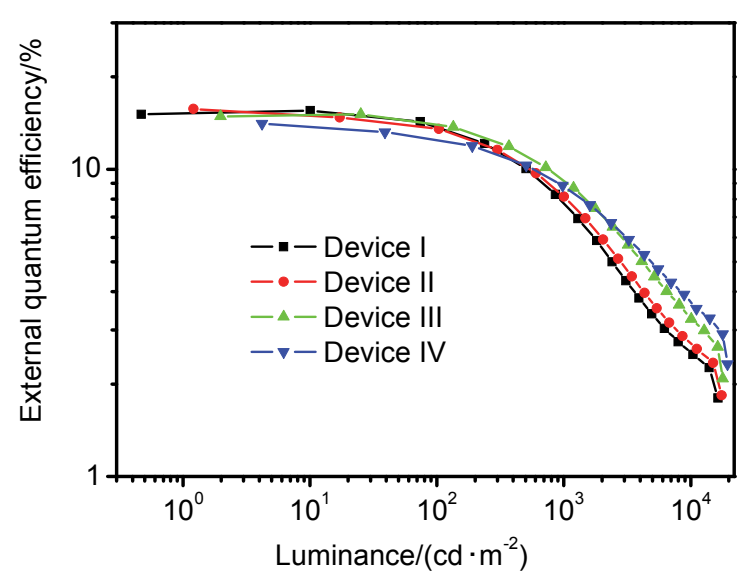

图 8 器件 I、II、III 和 IV 的外量子效率-亮度曲线

Figure 8 The external quantum efficiency-luminance characteristics of devices I, II, III and IV

物具有高的热稳定性和电化学稳定性. 理论计算显示, pPBPXZ 的 HOMO 主要分布在吩噁嗪结构单元上, 而 LUMO 主要分布在嘧啶环和苯环上, 两种分子轨道仅 有很小部分重叠. 由于 $\mathrm{pPBPXZ}$ 实现了 HOMO 与 LUMO 的互相分离, 其 $\Delta E_{\mathrm{ST}}$ 仅为 $0.09 \mathrm{eV}$, 充分满足了 $\mathrm{TADF}$ 材料的要求. 室温条件下, pPBPXZ 在甲苯溶液中 显示出了明显的 ICT 吸收和黄色荧光 $\left(\lambda_{\text {max }}=535 \mathrm{~nm}\right)$. 瞬态荧光衰减测试结果证实了 pPBPXZ 的室温发射光 中包含瞬时荧光和延迟苂光两种组分. 以 pPBPXZ 作为 发光层客体掺杂材料, 制备出了高效率的黄光电致发光 器件. 器件最大电流效率、功率效率和外量子效率分别 达到了 $49.9 \mathrm{~cd} / \mathrm{A} 、 49.0 \mathrm{~lm} / \mathrm{W}$ 和 $15.7 \%$, 而且器件发光效 率受 pPBPXZ 掺杂浓度影响较小, 说明该材料具有较高 的实际应用价值.

\section{4 实验部分}

\section{1 材料的合成}

\subsection{1 化合物 $\mathbf{3}$ 的合成}

将 $1.45 \mathrm{~g}(6 \mathrm{mmol}) 2$-氯-5-硒嘧啶、 $734 \mathrm{mg}(4 \mathrm{mmol})$ 吩噁嗪、 $768 \mathrm{mg}(8 \mathrm{mmol})$ 叔丁醇钠、 $44 \mathrm{mg}(0.2 \mathrm{mmol})$ 醋酸钯、 $174 \mathrm{mg}(0.6 \mathrm{mmol})$ 三叔丁基膦四氟硼酸盐和 30 $\mathrm{mL}$ 甲苯依次加入 $50 \mathrm{~mL}$ 烧瓶中, 氮气保护条件下 $95{ }^{\circ} \mathrm{C}$ 回流反应 $12 \mathrm{~h}$. 待反应液冷却至室温, 用硅藻土过滤, 滤液用旋转蒸发仪蒸干, 固体残留物用柱层析法提纯, 淋洗液为二氯甲烷和石油醚混合溶液 [ $V$ (二氯甲烷) : $V($ 石油醚 $)=1: 3$ ], 得到黄色粉末状产品 $652 \mathrm{mg}$, 产率 为 $55 \% .{ }^{1} \mathrm{H} \mathrm{NMR}\left(600 \mathrm{MHz}, \mathrm{CDCl}_{3}\right) \delta: 8.72(\mathrm{~s}, 2 \mathrm{H}), 6.78$ $(\mathrm{d}, J=4.2 \mathrm{~Hz}, 4 \mathrm{H}), 6.71 \sim 6.67(\mathrm{~m}, 2 \mathrm{H}), 5.94(\mathrm{~d}, J=7.8$ $\mathrm{Hz}, 2 \mathrm{H}) ;{ }^{13} \mathrm{C}$ NMR $\left(150 \mathrm{MHz}, \mathrm{CDCl}_{3}\right) \delta: 162.80,160.16$, $144.01,133.73,132.69,123.51,123.01,116.32,113.06$. MS (EI) $m / z$ : calcd for $\mathrm{C}_{16} \mathrm{H}_{10} \mathrm{ClN}_{3} \mathrm{O} 295.05$; found 295.27. Anal. calcd for $\mathrm{C}_{16} \mathrm{H}_{10} \mathrm{ClN}_{3} \mathrm{O}$ : C 64.98, H 3.41, N 14.21; found C 64.87, H 3.40, N 14.25.

\subsection{2 pPBPXZ 的合成}

将 $426 \mathrm{mg}$ (1.44 mmol)化合物 $3 、 120 \mathrm{mg}(0.72 \mathrm{mmol})$ 对苯二嗍酸、 $116 \mathrm{mg}(0.1 \mathrm{mmol}$ )四(三苯基膦)钯、 $10 \mathrm{~mL}$ 甲苯、 $5 \mathrm{~mL}$ 无水乙醇和 $2 \mathrm{~mL}$ 浓度为 $2 \mathrm{~mol} \cdot \mathrm{L}^{-1}$ 的 $\mathrm{K}_{2} \mathrm{CO}_{3}$ 水溶液依次加入 $50 \mathrm{~mL}$ 烧瓶中, 氮气保护条件下 $90{ }^{\circ} \mathrm{C}$ 回流反应 $12 \mathrm{~h}$. 待反应液冷却至室温, 用二氯甲烷萃取, 萃取液用无水 $\mathrm{MgSO}_{4}$ 干燥，然后用旋转蒸发仪蒸干，固 体残留物用柱层析法提纯, 淋洗液为二氯甲烷和石油醚 混合溶液 $[V($ 二氯甲烷 $): V($ 石油醚 $)=1: 3]$, 得到橙色 粉末状产品 $174 \mathrm{mg}$, 产率为 41\%. ${ }^{1} \mathrm{H} \mathrm{NMR} \mathrm{(600} \mathrm{MHz,}$ $\left.\mathrm{CDCl}_{3}\right) \delta: 8.91(\mathrm{~s}, 4 \mathrm{H}), 8.70(\mathrm{~s}, 4 \mathrm{H}), 6.80 \sim 6.74(\mathrm{~m}, 8 \mathrm{H})$, 6.68 (ddd, $J=7.9,7.0,1.9 \mathrm{~Hz}, 4 \mathrm{H}), 6.05$ (dd, $J=8.0,1.3$ $\mathrm{Hz}, 4 \mathrm{H}) ;{ }^{13} \mathrm{C} \mathrm{NMR}\left(150 \mathrm{MHz}, \mathrm{CDCl}_{3}\right) \delta: 163.24,160.39$, $145.63,144.02,139.04,133.23,132.63,128.88,123.48$, 122.58, 116.12, 113.08. MS (EI) $m / z$ : calcd for $\mathrm{C}_{38} \mathrm{H}_{24} \mathrm{~N}_{6} \mathrm{O}_{2}$ 596.20, found 596.45. Anal. calcd for $\mathrm{C}_{38} \mathrm{H}_{24} \mathrm{~N}_{6} \mathrm{O}_{2}$ : C 76.50, H 4.05, N 14.09; found C 76.41, H 4.03, N 14.04.

\section{2 电致发光器件的制备与性能测试}

将 ITO 玻璃基片(15 $\Omega$ )依次用无水乙醇、丙酮和去 离子水超声清洗 $5 \mathrm{~min}$, 然后 $120{ }^{\circ} \mathrm{C}$ 烘干, 最后用紫外 臭氧清洗仪处理 $10 \mathrm{~min}$. 真空条件下 $\left(5 \times 10^{-4} \mathrm{~Pa}\right)$, 首先 在清洗过的基片上蒸镀各有机层, 蒸镀速率为 $1 \sim 2 \AA / \mathrm{s}$; 然后, 蒸镀 $\mathrm{LiF}$ 和 $\mathrm{Al}$ 电极层, 蒸镀速率分别为 $1 \AA / \mathrm{s}$ 和 $10 \AA / s$. 电致发光光谱、亮度和 CIE 用 PR650 光谱光度 计测定, 电流密度和电压用 Keithley 2400 半导体特性测 试仪测定. 器件性能测试均由计算机控制, 在室温、常 压和空气环境中进行.

试剂、仪器、计算软件以及化合物核磁谱图见支持 信息(Supporting information).

\section{References}

[1] Sun, Y. R.; Giebink, N. C.; Kanno, H.; Ma, B. W.; Thompson, M. E.; Forrest, S. R. Nature 2006, 440, 908.

[2] Reineke, S.; Lindner, F.; Schwartz, G.; Seidler, N.; Walzer, K.; Lussem, B.; Leo, K. Nature 2009, 459, 234.

[3] Helander, M. G.; Wang, Z. B.; Qiu, J.; Greiner, M. T.; Puzzo, D. P.; Liu, Z. W. Science 2011, 332, 944.

[4] Han, T.-H.; Lee, Y.; Choi, M.-R.; Woo, S.-H.; Hong, B. H.; Ahn, J.-H.; Lee, T.-W. Nat. Photonics 2012, 459, 105.

[5] Sasabe, H.; Kido, J. J. Mater. Chem. C 2013, 1, 1699.

[6] Zhang, Z.; Li, W.; Ye, K.; Zhang, H. Acta Chim. Sinica 2016, 74, 179 (in Chinese). (张振宇, 李婉君, 叶开其, 张红雨, 化学学报, 2016, 74, 179.)

[7] Yu, Y.; Yang, J.; Ren, Z.; Xie, G.; Li, Q.; Li, Z. Acta Chim. Sinica 2016, 74, 865 .

[8] Wang, F.; Cao, X.; Mei, L.; Zhang, X.; Hu, J.; Tao, Y. Chinese J. Chem. 2018, 36, 241.

[9] Liang, X.; Wang, Z.; Wang, L.; Hanif, M.; Hu, D.; Su, S.; Xie, Z.; Gao, Y.; Yang, B.; Ma, Y. Chinese J. Chem. 2017, 35, 1559.

[10] Lin, D.; Song, S.; Chen, Z.; Guo, P.; Chen, J.; Shi, H.; Mai, Y.; Song, H. Chinese J. Org. Chem. 2018, 38, 103 (in Chinese). (林丹燕, 宋 森川, 陈智勇, 郭鹏然, 陈江韩, 史华红, 麦裕良, 宋化灿, 有机 化学, 2018, 38, 103.)

[11] Xu, H.; Chen, R.; Sun, Q.; Huang, W.; Liu, X. Chem. Soc. Rev. 2014, 43, 3259.

[12] Volz, D.; Wallesch, M.; Fléchon, C.; Danz, M.; Verma, A.; Navarro, 
J. M.; Zink, D. M.; Bräse, S.; Baumann, T. Green Chem. 2015, 17, 1988.

[13] Chi, Y.; Tong, B.; Chou, P.-T. Coord. Chem. Rev. 2014, 281, 1.

[14] Uoyama, H.; Goushi, K.; Shizu, K.; Nomura, H.; Adachi, C. Nature 2012, 492, 234.

[15] Tao, Y.; Yuan, K.; Chen, T.; Xu, P.; Li, H.; Chen, R.; Zheng, C.; Zhang, L.; Huang, W. Adv. Mater. 2014, 26, 7931.

[16] Kim, M.; Jeon, S. K.; Hwang, S. H.; Lee, S. S.; Yu, E.; Lee, J. Y. Chem. Commun. 2016, 52, 339.

[17] Lee, J.; Aizawa, N.; Yasuda, T. Chem. Mater. 2017, 29, 8012.

[18] Tamai, Y.; Ohkita, H.; Benten, H.; Ito, S.; Lee, J.-H.; Kido, J.; Park, J. J. Mater. Chem. C 2013, 1, 432.

[19] Chen, W.-C.; Lee, C.-S.; Tong, Q.-X. J. Mater. Chem. C 2015, 3, 10957.

[20] Chen, Y.-H.; Lin, C.-C.; Huang, M.-J.; Hung, K.; Wu, Y.-C.; Lin, W.-C.; Chen-Cheng, R.-W.; Lin, H.-W.; Cheng, C.-H. Chem. Sci. 2016, $7,4044$.

[21] Pan, Y.; Li, W.; Zhang, S.; Yao, L.; Gu, C.; Xu, H.; Yang, B.; Ma, Y. Adv. Opt. Mater. 2014, 2, 510.

[22] Konidena, R. K.; Thomas, K. R. J.; Dubey, D. K.; Sahoo, S.; Jou, J.-H. Chem. Commun. 2017, 53, 11802.

[23] Chen, W.-C.; Yuan, Y.; Ni, S.-F.; Tong, Q.-X.; Wong, F.-L.; Lee, C.-S. Chem. Sci. 2017, 8, 3599.

[24] Wang, Z.; Li, Y.; Cai, X.; Chen, D.; Xie, G.; Liu, K.; Wu, Y.; Lo, C.-C.; Lien, A.; Cao, Y.; Su, S.-J. ACS Appl. Mater. Interfaces 2016, 8,8627 .

[25] Chen, D.-Y.; Liu, W.; Zheng, C.-J.; Wang, K.; Li, F.; Tao, S.-L.; Ou, X.-M.; Zhang, X.-H. ACS Appl. Mater. Interfaces 2016, 8, 16791.

[26] Lee, D. R.; Choi, J. M.; Lee, C. W.; Lee, J. Y. ACS Appl. Mater. Interfaces 2016, 8, 23190.

[27] Cai, X.; Li, X.; Xie, G.; He, Z.; Gao, K.; Liu, K.; Chen, D.; Cao, Y.; Su, S.-J. Chem. Sci. 2016, 7, 4264.

[28] Li, F.; Xie, G.; Gong, S.; Wu, K.; Yang, C. Chem. Sci. 2016, 7, 5441.

[29] Rajamalli, P.; Senthilkumar, N.; Gandeepan, P.; Huang, P.-Y.;
Huang, M.-J.; Yang, C.-Y.; Chiu, M.-J.; Chu, L.-K.; Lin, H.-W.; Cheng, C.-H. J. Am. Chem. Soc. 2016, 138, 628.

[30] Liu, X.; Zhan, C.; Zheng, C.; Liu, C.; Lee, C.; Li, F.; Ou, X.; Zhang, X. Adv. Mater. 2015, 27, 2378.

[31] Wang, K.; Zheng, C. J.; Liu, W.; Liang, K.; Shi, Y. Z.; Tao, S. L.; Lee, C. S.; Ou, X. M.; Zhang, X. H. Adv. Mater. 2017, 29, 1701476.

[32] Yang, Z.; Mao, Z.; Xie, Z.; Zhang, Y.; Liu, S.; Zhao, J.; Xu, J.; Chi, Z.; Aldred, M. P. Soc. Rev. 2017, 46, 915.

[33] Liu, Y.; Li, C.; Ren, Z.; Yan, S.; Bryce, M. R. Nat. Rev. Mater. 2018 , 3, 18020 .

[34] Wong, M. Y.; Zysman-Colman, E. Adv. Mater. 2017, 29, 1605444.

[35] Xiang, Y.; Gong, S.; Zhao, Y.; Yin, X.; Luo, J.; Wu, K.; Lu, Z.; Yang, C. J. Mater. Chem. C 2016, 4, 9998.

[36] Xie, G.; Li, X.; Chen, D.; Wang, Z.; Cai, X.; Chen, D.; Li, Y.; Liu, K.; Cao, Y.; Su, S. J. Adv. Mater. 2016, 28, 181 .

[37] Chen, J.-X.; Wang, K.; Zheng, C.-J.; Zhang, M.; Shi, Y.-Z.; Tao, S.-L.; Lin, H.; Liu, W.; Tao, W.-W.; Ou, X.-M.; Zhang, X.-H. $A d v$. Sci. 2018, 5, 1800436.

[38] Chen, X.; Yang, Z.; Xie, Z.; Zhao, J.; Yang, Z.; Aldred, M. P.; Chi, Z. Mater. Chem. Front. 2018, 2, 1017.

[39] Chen, Y.; Li, S.; Hu, T.; Wei, X.; Li, Z.; Liu, W.; Liu, J.; Wang, R.; Yi, Y.; Zhao, C.; Wang, Y.; Wang, P. J. Mater. Chem. C 2018, 6, 2951.

[40] Xiang, Y.; Zhu, Z.-L.; Xie, D.; Gong, S.; Wu, K.; Xie, G.; Lee, C.-S.; Yang, C. J. Mater. Chem. C 2018, 6, 7111.

[41] Huang, J.; Nie, H.; Zeng, J.; Zhuang, Z.; Gan, S.; Cai, Y.; Guo, J.; $\mathrm{Su}$, S.-J. Angew. Chem. Int. Ed. 2017, 56, 12971.

[42] Li, J.; Ding, D. X.; Tao, Y. T.; Wei, Y. Y.; Chen, R. F.; Xie, L. H.; Haung, W.; Xu, H. Adv. Mater. 2016, $28,3122$.

[43] Pan, K.-C.; Li, S.-W.; Ho, Y.-Y.; Shiu, Y.-J.; Tsai, W.-L.; Jiao, M.; Lee, W.-K.; Wu, C.-C.; Chung, C.-L.; Chatterjee, T.; Li, Y.-S.; Wong, K.-T.; Hu, H.-C.; Chen, C.-C.; Lee, M.-T. Adv. Funct. Mater 2016, 26, 7560 . 